\title{
Improvement in PET/CT image quality in overweight patients with PSF and TOF
}

\author{
Takafumi Taniguchi · Go Akamatsu • Yukiko Kasahara - Katsuhiko Mitsumoto • \\ Shingo Baba $\cdot$ Yuji Tsutsui $\cdot$ Kazuhiko Himuro $\cdot$ Shohei Mikasa • \\ Daisuke Kidera $\cdot$ Masayuki Sasaki
}

Received: 20 April 2014/ Accepted: 19 September 2014/Published online: 26 September 2014

(C) The Japanese Society of Nuclear Medicine 2014

\begin{abstract}
Objective The aim of this study was to evaluate the effect of the point spread function (PSF) and time of flight (TOF) on PET/CT images of overweight patients in relation to the iteration number and the acquisition time.

Methods This study consisted of a phantom study and a clinical study. The NEMA IEC body phantom and a $40 \mathrm{~cm}$ diameter large phantom (LG phantom) simulating an overweight patient were used in this study. Both phantoms were filled with ${ }^{18} \mathrm{~F}$ solution with a sphere to background ratio of 4:1. The PET data were reconstructed with the baseline ordered-subsets expectation maximization (OSEM) algorithm, with the OSEM + PSF model, with the OSEM + TOF model and with the OSEM + PSF + TOF model. The clinical study was a retrospective analysis of 66 patients who underwent ${ }^{18} \mathrm{~F}$-FDG PET/CT. The image quality was evaluated using the background variability (coefficient of variance, $\mathrm{CV}_{\text {phantom }}$ and $\left.\mathrm{CV}_{\text {liver }}\right)$ and the contrast $\left(\mathrm{CONT}_{\mathrm{HOT}}\right.$ and SNR).

Results In phantom study, the $\mathrm{CV}_{\text {phantom }}$ of the $\mathrm{LG}$ phantom was higher than that of the NEMA phantom. The
\end{abstract}

T. Taniguchi · G. Akamatsu · Y. Kasahara - K. Mitsumoto ·

S. Mikasa · D. Kidera · M. Sasaki $(\bowtie)$

Division of Medical Quantum Science, Department of Health

Sciences, Graduate School of Medical Sciences, Kyushu

University, 3-1-1 Maidashi, Higashi-ku, Fukuoka 812-8582,

Japan

e-mail: msasaki@hs.med.kyushu-u.ac.jp

S. Baba

Department of Clinical Radiology, Graduate School of Medical

Sciences, Kyushu University, Fukuoka, Japan

Y. Tsutsui · K. Himuro

Department of Medical Technology, Kyushu University

Hospital, Fukuoka, Japan
PSF decreased the $\mathrm{CV}_{\text {phantom }}$ of the LG phantom to the NEMA phantom level. The TOF information accelerated the $\mathrm{CV}_{\text {phantom }}$ plateau earlier. The best relationship between the $\mathrm{CV}_{\text {phantom }}$ and the $\mathrm{CONT}_{\mathrm{HOT}}$ was observed for the OSEM + PSF + TOF. In clinical study, the combination of PSF and TOF decreased the $\mathrm{CV}_{\text {liver }}$ for overweight patients to that for normal weight patients while it increased the SNR similarly between two patient groups.

Conclusion The combination of the PSF and TOF correction improved the image quality of the LG phantom and overweight patients.

Keywords Point-spread-function - Time-of-flight · PET/ $\mathrm{CT} \cdot$ Image quality $\cdot$ Overweight

\section{Introduction}

${ }^{18}$ F-fluoro 2-deoxy-D-glucose (FDG) positron emission tomography (PET) is useful for diagnosis, tumor staging, monitoring of response to therapy and for prediction of prognosis in a variety of tumors [1-3]. The image quality of $\mathrm{PET} / \mathrm{CT}$ images is important to correctly diagnose cancer [4]. However, the PET image quality of overweight patients is often poor resulting in increased image noise because of the increase in photon attenuation and the scatter fraction [5]. Such images are sometimes difficult to be diagnosed with lower confidence of the reader [6]. For improving the image quality in such patients, either an increase of the injected dose of radiopharmaceuticals or an elongation of acquisition times is generally considered to be important. However, the increase of the injected dose of FDG is difficult in some institutions when the commercially distributed FDG is used. Therefore, the acquisition time has been reported to be an especially important parameter [6, 7]. However, the 
extension of the acquisition time is burden for patients and is also inefficient for institutions.

Recently, the point spread function (PSF) correction and time of flight (TOF) information were incorporated into the reconstruction algorithm [8]. The PSF information measured in a large number of positions in the field of view (FOV) is incorporated into reconstructed algorithms. Therefore, the PSF correction is expected to improve the spatial resolution and to reduce distortions [9]. On the other hand, the TOF includes the time information to correctly recognize the localization of annihilation points along the line-of-responses (LORs). Therefore, TOF information is considered to reduce the noise and to improve the contrast $[10,11]$. Thus, both the PSF and TOF information are expected to improve the image quality for overweight patients. The usefulness of TOF information on improving an image quality of overweight patients have been examined using large diameter phantoms $[12,13]$. TOF information was also reported on bringing the image quality of overweight patients closer to that of normal weight patients in a clinical study [12-14]. By using a combination of the PSF and TOF information, we have already reported an improvement of image quality in patients with high body mass index clinically $[15,16]$. However, few reports have examined the effect of combined PSF and TOF information in a combined phantom and clinical examination in relation to the body weight.

The aim of this study was to investigate the effects of the PSF and TOF on PET/CT images using an originally designed large body phantom simulating overweight patients and actual overweight patients in the clinical setting.

\section{Materials and methods}

\section{Phantoms}

A National Electrical Manufacturers Association (NEMA) International Electrotechnical Commission (IEC) body phantom (NEMA phantom) and a $40 \mathrm{~cm}$ diameter large phantom (LG phantom) were used in this study. The length of major axis of the NEMA phantom was $30 \mathrm{~cm}$. The inner diameter of the hot spheres was from 10 to $37 \mathrm{~mm}(10,13$, 17, 22, 28 and $37 \mathrm{~mm}$ ).

The LG phantom was originally designed for this study (Chiyoda Technol, Tokyo, Japan and YAMATO Jushi Inc, Tokyo, Japan) (Fig. 1). The diameters of the outer and inner layers were 40 and $34 \mathrm{~cm}$, respectively. The thickness of the outer layer was $2 \mathrm{~cm}$. The circumference was $126 \mathrm{~cm}$, which is compatible to the waist of patients simulating about a $100 \mathrm{~kg}$ body weight person [17].

The LG phantom with NEMA phantom simulated an overweight subject where the LG phantom for soft tissue, background of NEMA phantom for liver and hot spheres in NEMA phantom for lesions. The radioactivity in each compartment was $2.65,7.95$ and $31.8 \mathrm{kBq} / \mathrm{mL}$, respectively (proportion, 1:3:12).

\section{Patients}

Sixty-six patients were retrospectively analyzed, consisting of 36 males and 30 females. The mean age was $62.3 \pm 13.1$ years (range 34-85 years). The clinical indication for the FDG PET/CT examination of these patients were the evaluation of lung cancer $(n=11)$, general cancer screening $(n=9)$, thyroid cancer $(n=8)$, malignant lymphoma $(n=8)$, colorectal cancer $(n=7)$, head and neck cancer $(n=6)$, gastric cancer $(n=3)$, cervical cancer $(n=2)$, breast cancer $(n=2)$, esophageal carcinoma $(n=2)$, malignant mesothelioma $(n=1)$, glioblastoma $(n=1)$, lung metastasis $(n=1)$, pneumonia $(n=1)$, cholangiocarcinoma $(n=1)$, melanoma $(n=1)$, pancreatic cancer $(n=1)$ and unknown primary cancer $(n=1)$.

The mean body weight was $59.9 \pm 13.5 \mathrm{~kg}$ (range $35-103 \mathrm{~kg}$ ). The patients were classified into 2 groups based on their weight; normal weight, $<70 \mathrm{~kg}(n=48$, average $53.5 \pm 8.35 \mathrm{~kg})$ and overweight, $\geq 70 \mathrm{~kg}(n=18$, average $77.0 \pm 8.56 \mathrm{~kg}$ ).

In 40 lesions in 24 patients, histopathologically confirmed malignant lesions were analyzed for the contrast of tumor FDG uptake. Twenty-eight lesions were in 18 patients with normal weight group and 12 lesions in 6 patients with overweight group. The long axis diameter of lesions was $10.7 \pm 0.5 \mathrm{~mm}$ (range 5.8-30.0 mm).
Fig. 1 The LG phantom loaded with the NEMA phantom. The blueprint of the LG phantom viewed from top and from the left side (a), and a photograph of the phantom (b)

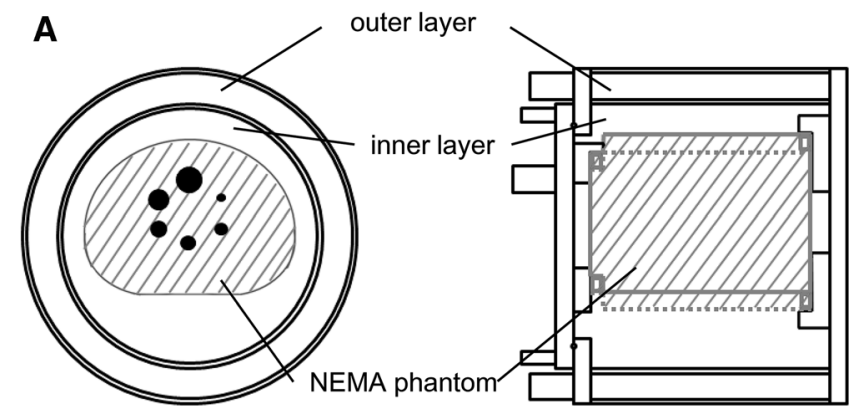

B

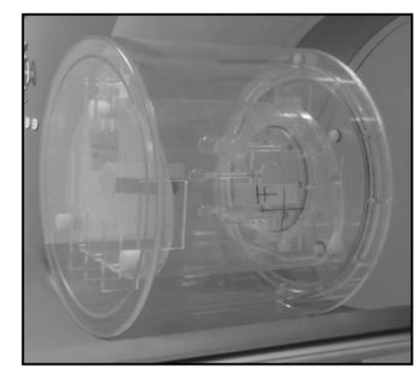


This clinical study was approved by the ethics committee of our institution. This study was retrospective in nature, and its results did not influence any further therapeutic decision-making.

\section{PET/CT protocol}

All data were acquired using the Biograph mCT scanner (Siemens Healthcare, Erlangen, Germany). The system timing resolution was $555 \mathrm{ps}$ and the coincidence timing window was $4.1 \mathrm{~ns}$. The crystal was combined lutetium oxyorthosilicate (LSO) detectors $\left(4 \times 4 \times 20 \mathrm{~mm}^{3}\right)$. The number of blocks per detector ring was 48 and the total number of blocks was 144 . The system sensitivity was $5.4 \mathrm{cps} / \mathrm{kBq}$ [18]. Scanning parameters for CT were as follows: $120 \mathrm{kV}, 100 \mathrm{~mA}, 5-\mathrm{mm}$ slice thickness, $0.5 \mathrm{~s}$ rotation. The CT data were used for the attenuation correction.

The phantom data acquisition was performed in 3-dimensional (3D) mode for $20 \mathrm{~min}$ in listmode. On the other hand, the clinical data acquisition was performed in whole body $3 \mathrm{D}$ mode for 2 min per bed position with an overlap of $50 \%$. All patients fasted for at least four hours before FDG administration. The patients with high fasting blood sugar (blood sugar $\geq 150 \mathrm{mg} / \mathrm{dL}$ ) were excluded. Emission scans from the midthigh to the top of the head were started at $1 \mathrm{~h}$ after injection of $226.2 \pm 49.3 \mathrm{MBq}$ (range 135.9-317.5 MBq) of FDG (Nihon Medi-Physics Co., Ltd., Tokyo, Japan). The injected dose was $3.97 \pm 1.26 \mathrm{MBq} / \mathrm{kg}$.

The PET data were then reconstructed by the ordered subsets expectation maximization (OSEM) algorithm, the OSEM with PSF correction, the OSEM with TOF information and the OSEM with both PSF and TOF information. For a phantom study, the number of iterations varied from 1 to 10 , and the acquisition times varied from 1 to $10 \mathrm{~min}$. The number of subsets was 24 for the reconstructions without TOF and 21 for those with TOF. The image matrix was $256 \times 256$. No smoothing filter was used for postprocessing. For a clinical study, the reconstruction parameters were 2 iteration and 21 subset for the algorithms with TOF and 3 iteration and 24 subset for the algorithms without TOF [15]. The Gaussian filter was used as the smoothing filter, and the full width half maximum was $2 \mathrm{~mm}$ for the algorithms with PSF and $4 \mathrm{~mm}$ for the algorithms without PSF [15]. The image matrix was $256 \times 256$.

Imaging analysis

\section{Phantom study}

Since the $10 \mathrm{~mm}$ hot sphere was not visually recognized in some images, the quantitative analysis was performed using a $13 \mathrm{~mm}$ hot sphere. The visibility of hot spheres was assessed by a nuclear medicine physician and 2 radiological technologists. The visibility was classified into 3 grades (2: identifiable, 1: visualized but similar hot spots were observed elsewhere, 0: not visualized). A score of 1.5 is considered to represent a clinically interpretable study [19].

The PET image analyses were performed based on the contrast of a $13 \mathrm{~mm}$ hot sphere $\left(\mathrm{CONT}_{13 \mathrm{~mm}}\right)$ and the coefficient of variance of the background $\left(\mathrm{CV}_{\text {phantom }}\right)$. On the hot spheres, the regions of interest (ROIs) were drawn in the slice of the sphere center. The diameters of the ROIs were the same as the inner diameter of each sphere. On the background, 12 ROIs of $30 \mathrm{~mm}$ in diameter were drawn in the central slice of the body phantom and in slices that were $\pm 5 \mathrm{~mm}$ apart (total $36 \mathrm{ROIs}$ ).

$$
\begin{aligned}
& \mathrm{CONT}_{13 \mathrm{~mm}}=\mathrm{HOT} / \mathrm{BG}_{\text {phantom }} \\
& \mathrm{CV}_{\text {phantom }}=\mathrm{SD}_{\text {phantom }} / \mathrm{BG}_{\text {phantom }} \times 100(\%)
\end{aligned}
$$

where the HOT was the average activity in the $13 \mathrm{~mm}$ hot sphere ROI, the $\mathrm{BG}_{\text {phantom }}$ was the average activity in the 36 background ROIs and the $\mathrm{SD}_{\text {phantom }}$ was the standard deviation of the 36 background ROIs.

\section{Clinical study}

The quality of clinical PET images was analyzed by the contrast of tumors $\left(\mathrm{CONT}_{\text {tumor }}\right)$, the $\mathrm{CV}$ of the liver $\left(\mathrm{CV}_{\text {liver }}\right)$ and signal-to-noise ratio (SNR). For tumor ROIs, tumor boundaries on CT images were manually traced and placed on PET images. The 3 circular ROIs with $30 \mathrm{~mm}$ in diameter were placed on 3 contiguous axial slices in the largest liver section. The ROIs were carefully placed in a uniform area of the liver so as not to include the portal vein or the hepatic hilum.

$$
\begin{aligned}
& \mathrm{CONT}_{\text {tumor }}=\mathrm{TUMOR} / \mathrm{BG}_{\text {liver }} \\
& \mathrm{CV}_{\text {liver }}=\mathrm{SD}_{\text {liver }} / \mathrm{BG}_{\text {liver }} \times 100(\%) \\
& \mathrm{SNR}=\mathrm{CONT}_{\text {tumor }} / \mathrm{CV}_{\text {liver }}
\end{aligned}
$$

where the TUMOR was the average activity in the tumor $\mathrm{ROI}$, the $\mathrm{SD}_{\text {liver }}$ was the standard deviation and the $\mathrm{BG}_{\text {liver }}$ was the mean activity of the 9 liver ROIs.

Statistical analysis

The $\mathrm{CV}_{\text {phantom }}, \mathrm{CV}_{\text {liver }}, \mathrm{CONT}_{\text {tumor }}$ and $\mathrm{SNR}$ of each reconstruction method were compared using paired $t$ test, Wilcoxon signed-rank tests and Student's $t$ test. In this analysis, $P<0.05$ was considered to indicate statistical significance.

\section{Results}

Phantom study

Figure 2 shows the PET images of both the NEMA phantom and the LG phantom reconstructed by 4 
different algorithms. The images of both the NEMA phantom and the LG phantom exhibited increased noise by the increase of iteration number (Fig. 2a). The LG phantom was noisier than the NEMA phantom. At iteration 2 of the LG phantom, the $13 \mathrm{~mm}$ hot sphere was not clearly observed when reconstructed by OSEM and the OSEM + PSF, while it was clearly recognized by the OSEM + TOF and the OSEM + PSF + TOF. In contrast, the $13 \mathrm{~mm}$ hot sphere in the NEMA phantom was clearly observed in all reconstruction models. At 2 min or longer acquisition time, all hot spheres of the NEMA phantom were clearly observed in all reconstruction models (Fig. 2b). However, the $10 \mathrm{~mm}$ hot sphere in the LG phantom was not observed even at $10 \mathrm{~min}$ acquisition.

The $\mathrm{CV}_{\text {phantom }}$ was examined in relation to the iteration number (Fig. 3a). In both the NEMA phantom and the LG phantom, the $\mathrm{CV}_{\text {phantom }}$ increased with the iteration number increased. The $\mathrm{CV}_{\text {phantom }}$ of the $\mathrm{LG}$ phantom was about twice of that of the NEMA phantom. In the OSEM + TOF, the $\mathrm{CV}_{\text {phantom }}$ plateaued earlier. On the other hand, the $\mathrm{CV}_{\text {phantom }}$ was relatively low in images reconstructed with

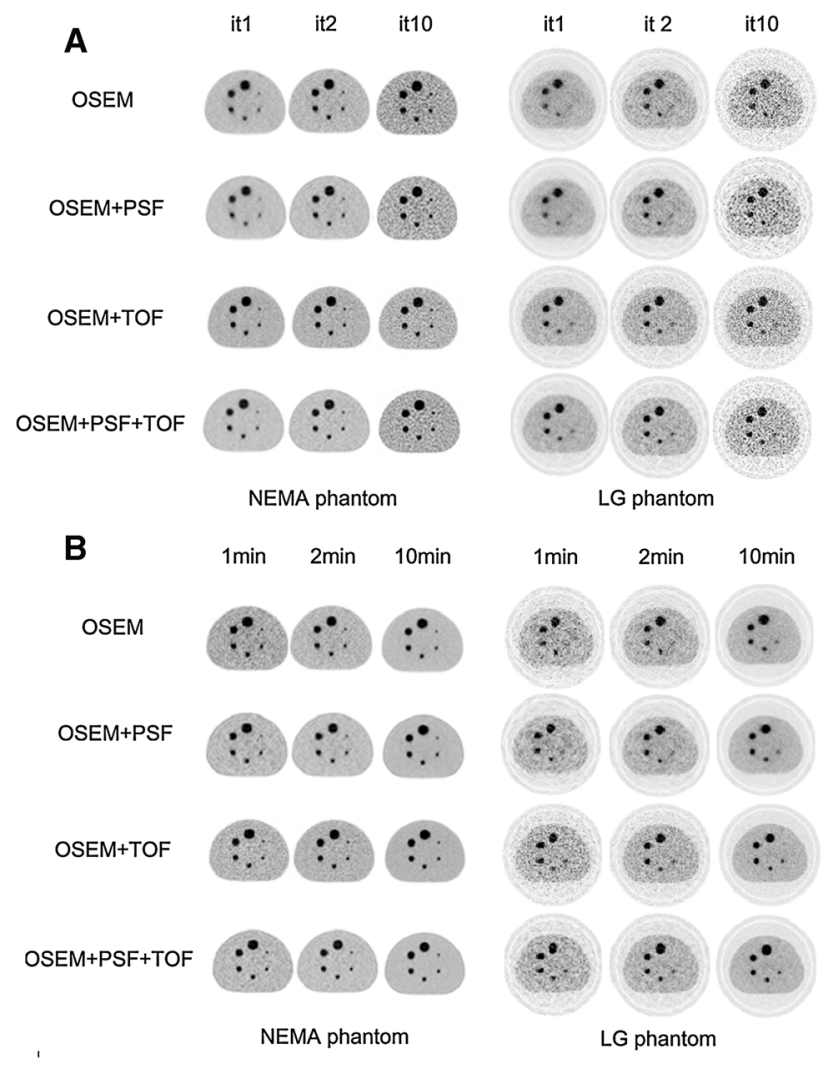

Fig. 2 The PET images with different reconstruction algorithms in relation to the iteration numbers (a) and in relation to the acquisition times (b). The image quality of the NEMA phantom was superior to that of the LG phantom for all iteration numbers and acquisition times
PSF (both the OSEM + PSF and the OSEM + PSF + TOF). In Fig. $3 b$, the $\mathrm{CV}_{\text {phantom }}$ was examined in relation to the acquisition time. In the NEMA phantom, the $\mathrm{CV}_{\text {phantom }}$ of $10 \%$ or lower was obtained for $2 \mathrm{~min}$ or longer acquisition time in images reconstructed with PSF (the OSEM + PSF and the OSEM + PSF + TOF). Although the $\mathrm{CV}_{\text {phantom }}$ of the LG phantom with OSEM was not below $10 \%$ within 10 min acquisition, a $10 \%$ or lower $\mathrm{CV}_{\text {phantom }}$ was obtained at $6-8 \mathrm{~min}$ in the $\mathrm{LG}$ phantom with PSF.

The relationship between the $\mathrm{CV}_{\text {phantom and the }}$ $\mathrm{CONT}_{13 \mathrm{~mm}}$ was shown in Fig. 4. The $x$-axis was the $\mathrm{CV}_{\text {phantom }}$ and the $y$-axis was the $\mathrm{CONT}_{13 \mathrm{~mm}}$, and each plot means the iteration number. A curve exist closer to the upper left corner meant that high contrast was obtained at a

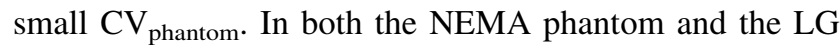
phantom, the curve of the OSEM + PSF and the OSEM + PSF + TOF were near the upper left corner. The best relationship in the LG phantom was observed for the OSEM + PSF + TOF. Furthermore, for the LG phantom, the $\mathrm{CONT}_{13 \mathrm{~mm}}$ obtained already at the 2 iteration with the OSEM + PSF + TOF could not be reached by other reconstruction methods at any iteration (up to 10). This clearly indicates the advantage of TOF reconstruction for large objects and possibility patients.

\section{Clinical study}

The $\mathrm{CV}_{\text {liver }}$ of the patients in relation to the body weight was shown in Fig. 5. In both the normal and overweight groups, the $\mathrm{CV}_{\text {liver }}$ was significantly low in images reconstructed with TOF $(P<0.001$ for the normal group, $P<0.001$ for the overweight group). Although those with PSF were significantly low in the overweight group $(P=0.009)$, those were not low in the normal weight group $(P=0.335)$. The lowest $\mathrm{CV}_{\text {liver }}$ was observed in images reconstructed with OSEM $+\mathrm{PSF}+\mathrm{TOF}$ ( $P<0.001$ for the normal group, $P<0.001$ for the overweight group). The $\mathrm{CV}_{\text {liver }}$ with the OSEM $+\mathrm{PSF}+\mathrm{TOF}$ for the overweight group was almost equal to that of the OSEM for the normal weight group $(P=0.579)$.

The SNR in relation to the body weight was shown in Fig. 6. In both normal and overweight groups, the SNR was significantly increased by PSF $(P<0.001$ for the normal group, $P=0.001$ for the overweight group) and by TOF ( $P<0.001$ for the normal group, $P=0.001$ for the overweight group). The highest SNR was observed in images reconstructed with OSEM $+\mathrm{PSF}+\mathrm{TOF}$ ( $P<0.001$ for the normal group, $P<0.001$ for the overweight group). The degree of improvement in SNR for the overweight group was almost equal to that for the normal weight group. 
Fig. 3 The $\mathrm{CV}_{\text {phantom }}$ of the NEMA phantom and LG function of the acquisition time (b) (a 2 min acquisition, b 2 iteration). The $\mathrm{CV}_{\text {phantom }}$ of the LG phantom was almost twice as high as that of the NEMA phantom. The $\mathrm{CV}_{\text {phantom was }}$ lower when the PSF information was incorporated

$(\mathrm{OSEM}+\mathrm{PSF}$ and

$\mathrm{OSEM}+\mathrm{PSF}+\mathrm{TOF})$.

$\mathrm{A} \mathrm{CV}_{\text {phantom }}$ of $10 \%$ or lower was obtained for 2 min or longer acquisition time in information phantom as a function of the iteration number (a) and as a images reconstructed with PSF
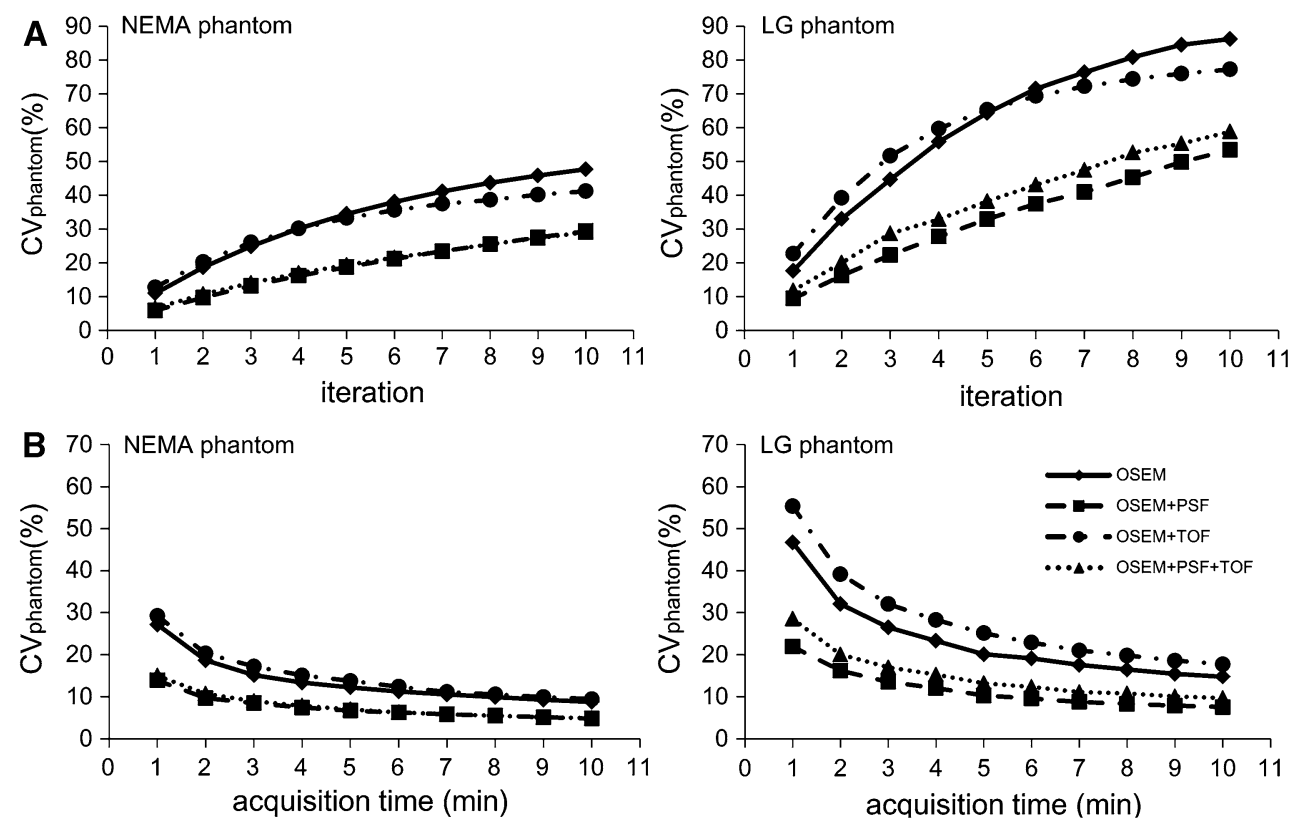

Fig. 4 The relationship between the $\mathrm{CV}_{\text {phantom }}$ and the CONT for the $2 \mathrm{~min}$ acquisition. a The NEMA phantom and $\mathbf{b}$ the LG phantom. Each plot means the iteration number. In both phantoms, the curves of the OSEM + PSF and the OSEM + PSF + TOF were closer to the upper left corner

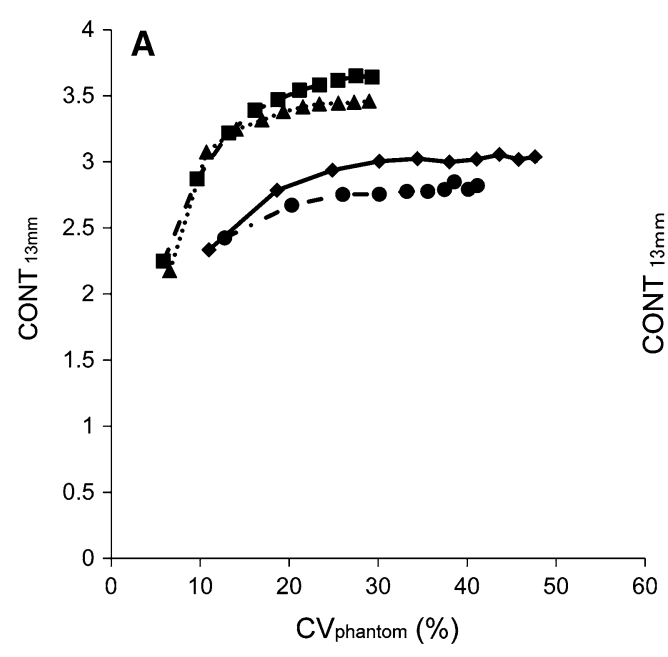

\section{Discussion}

In this study, we developed a large body phantom to examine the effects of the PSF and TOF on the PET image quality for simulated overweight subjects. The $\mathrm{CV}_{\text {phantom }}$ of the LG phantom was higher than that of the NEMA phantom. Both a good $\mathrm{CONT}_{13 \mathrm{~mm}}$ and $\mathrm{CV}_{\text {phantom }}$ of the $\mathrm{LG}$ phantom were obtained with a short acquisition time using the OSEM + PSF + TOF. The $\mathrm{CV}_{\text {liver }}$ in the overweight patients was decreased by the OSEM + PSF + TOF. The SNR was similarly increased between two patient groups.

The CV is known to vary with body weight [20]. The NEMA phantom represents standard body size patients simulating patients with a $60 \mathrm{~kg}$ body weight and $84 \mathrm{~cm}$ waist [21]. In the present study, we originally designed a large phantom simulating patients with a body weight of approximately $100 \mathrm{~kg}$ and a waist circumference of $126 \mathrm{~cm}$ [17]. Nagaki et al. [20] observed that the noise of overweight patients was larger than that of normal weight patients. They reported that the $\mathrm{CV}_{\text {liver }}$ of PET images for 60-74 kg patients was $10.98 \%$, and that for patients over $75 \mathrm{~kg}$ was $14.15 \%$. Our results showed that the $\mathrm{CV}_{\text {phantom }}$ of the LG phantom was almost twice of that of the NEMA phantom.

The $\mathrm{CV}_{\text {phantom }}$ below $10 \%$ was not obtained in the $\mathrm{LG}$ phantom within $10 \mathrm{~min}$ for the OSEM. To obtain the $\mathrm{CV}_{\text {phantom }}$ of $20 \%$ or less, an almost 2.5 times longer acquisition time was necessary in the LG phantom in 


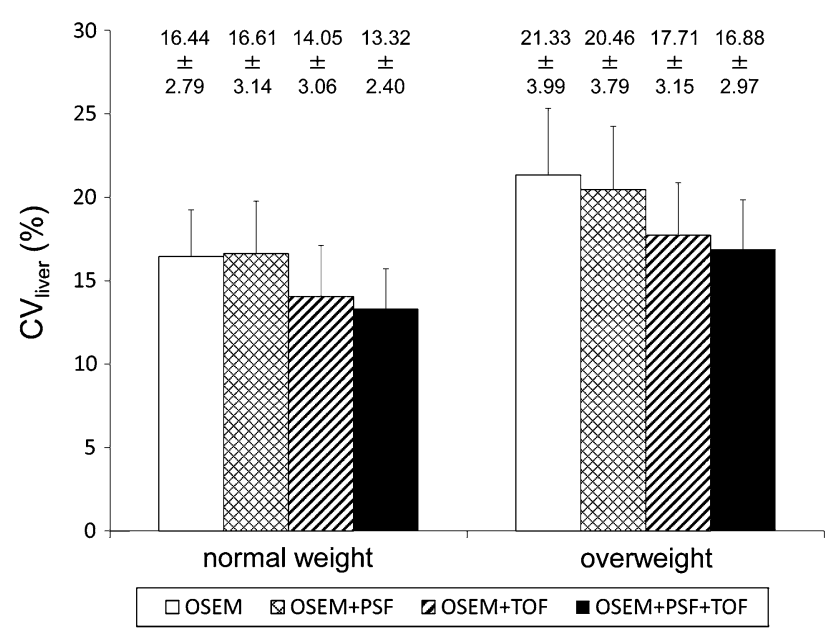

Fig. 5 The $\mathrm{CV}_{\text {liver }}$ of normal weight patients and overweight patients. The $\mathrm{CV}_{\text {liver }}$ was low in images reconstructed with TOF information (OSEM + TOF and OSEM + PSF + TOF). The $\mathrm{CV}_{\text {liver }}$ with the OSEM + PSF + TOF for the overweight group was almost equal to that with the OSEM for the normal weight group (*significantly different from both OSEM and OSEM + PSF)

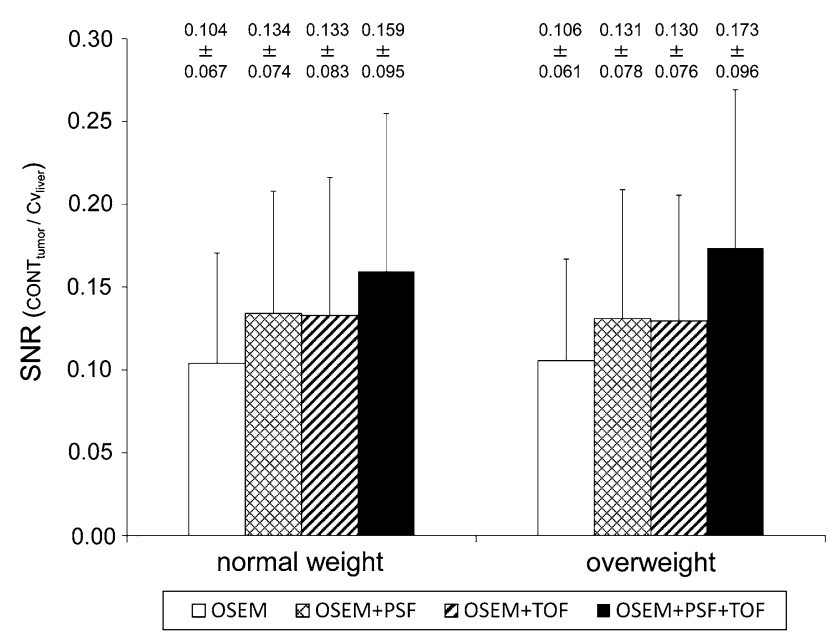

Fig. 6 The SNR of normal weight patients and overweight patients. The SNR was significantly increased by PSF and by TOF. The degree of improvement was not different between the normal and overweight patients

comparison with the NEMA phantom. On the other hand, the $\mathrm{CV}_{\text {phantom }}$ under $10 \%$ was obtained in the $\mathrm{LG}$ phantom with PSF correction. PET image reconstructed with the PSF correction showed a more uniform background than the image reconstructed without the PSF correction. Moreover, the contrast and CV have a trade-off relationship, and the PSF correction slows the iterative convergence. Therefore, the PSF correction was considered to reduce the $\mathrm{CV}_{\text {phantom [15]. However, a 3-4 times longer }}$ acquisition time was necessary to obtain the same $\mathrm{CV}_{\text {phantom }}$ of the NEMA phantom. A previous study reported that the optimal acquisition time for overweight patients was about 1.6 times longer than that for normal weight patients [20]. The difference between the studies was considered to be due to the weight of the objects. The LG phantom in our study simulated a $100 \mathrm{~kg}$ object, while the average body weight of their study was approximately $85 \mathrm{~kg}$. Both studies demonstrated that obtaining high quality PET images of overweight patients requires a longer acquisition time than that of normal weight patients.

According to the relationship between the $\mathrm{CV}_{\text {phantom }}$ and the $\mathrm{CONT}_{13 \mathrm{~mm}}$, the quality of PET images reconstructed with the OSEM + PSF and the OSEM + PSF + TOF was superior to that of the OSEM for both in the NEMA and LG phantoms. The $\mathrm{CONT}_{13 \mathrm{~mm}}$ was markedly improved for the OSEM + PSF + TOF with a smaller iteration number. This improvement is the effect of the TOF information, which accelerates the convergence of the $\mathrm{CONT}_{13 \mathrm{~mm}}$ in comparison with the algorithms without TOF [22]. A previous report using the phantom that was $35 \mathrm{~cm}$ in diameter showed a high contrast even for the faster convergence of the OSEM + PSF + TOF for a $13 \mathrm{~mm}$ sphere [12]. Surti et al. [13] also reported that the contrast with TOF information was about 2-fold compared with that without TOF information. These findings suggested that the addition of PSF and TOF information to the iterative reconstruction algorithm improved both noise and contrast in PET images.

Clinically, the $\mathrm{CV}_{\text {liver }}$ improved after the PSF and TOF information in this study. In particular, the value with the OSEM + PSF + TOF for overweight patients was almost equal to that with the OSEM for normal weight patients. Our previous study showed that the PSF and TOF information improved the image quality of patients with a high body mass index (BMI) [15, 16]. Karp et al. [23] reported that the PET images with TOF information had high contrast, faster convergence and low noise in large patients. Thus, the TOF information was expected to enhance the detectability of lesions in overweight patients with a smaller iteration number. These studies suggested that both PSF and TOF information are considered to improve the image quality and lesion detectability.

The SNR was improved on images reconstructed by using PSF and TOF information. The best improvement was obtained by the combination of PSF and TOF information (OSEM + PSF + TOF). The degree of improvement of the SNR was not different between the normal and overweight groups. The improvement of spatial resolution by PSF is considered to lead to an increase in the SNR [24]. Furthermore, our previous study suggested the combination of PSF and TOF has the potential to maintain image quality for low activity objects and a shorter acquisition time [15]. Thus, our results suggest that the effect of PSF and TOF information on the improvement of the SNR was similar between the normal and overweight groups. 
This study had some limitations. First, we examined only one phantom simulating a patient with $100 \mathrm{~kg}$ body weight. A phantom study using some different sizes of phantoms may be required. Second, the LG phantom used in the study is circular though the body shape of most subject is elliptical. Third, the number of patients heavier than $100 \mathrm{~kg}$ was limited in our study. Further examinations with such patients are expected to enhance the advantages of the PSF and TOF information for overweight patients. Fourth, we did not evaluate the lesion detectability. Fakhri et al. reported that the effect of TOF information was large for higher BMI. Therefore the overweight patients were considered to show the great effect of TOF information [19, 25]. Clinical examinations to assess the lesion detectability in overweight patients will be necessary to confirm the clinical usefulness of the PSF and TOF information.

\section{Conclusion}

In conclusion, our results indicated that the OSEM + PSF + TOF improved the PET images of a LG phantom, and is thus considered to be useful for achieving good image quality in overweight patients.

Acknowledgments The authors thank the staff of the Department of Clinical Radiology and Medical Technology at Kyushu University Hospital for their valuable clinical support. No potential conflicts of interest were disclosed.

\section{References}

1. Coleman RE. PET in lung cancer. J Nucl Med. 1999;40:814-20.

2. Delbeke D. Oncological applications of FDG PET imaging: brain tumors, colorectal cancer lymphoma and melanoma. J Nucl Med. 1999;40:591-603.

3. Gambhir SS, Czernin J, Schwimmer J, Silverman DS, Coleman RE, Phelps ME, et al. A tabulated summary of the FDG PET literature. J Nucl Med. 2001;42:1S-93S.

4. Masuda Y, Kondo C, Matsuo Y, Uetani M, Kusakabe K. Comparison of imaging protocols for ${ }^{18} \mathrm{~F}-\mathrm{FDG}$ PET/CT in overweight patients: optimizing scan duration versus administered dose. J Nucl Med. 2009;50:844-8.

5. Halpern BS, Dahlbom M, Auerbach MA, Schiepers C, Fueger BJ, Weber WA, et al. Optimizing imaging protocols for overweight and obese patients: a lutetium orthosilicate PET/CT study. J Nucl Med. 2005;46:603-7.

6. Tatsumi M, Clark PA, Nakamoto Y, Wahl RL. Impact of body habitus on quantitative and qualitative image quality in wholebody FDG-PET. Eur J Nucl Med. 2003;30:40-5.

7. Halpern BS, Dahlbom M, Quan A, Schiepers C, Waldherr C, Silverman DH, et al. Impact of patient weight and emission scan duration on PET/CT image quality and lesion detectability. J Nucl Med. 2004;45:797-801.

8. Marti-Climent JM, Prieto E, Dominguez-Prado I, Garcia-Velloso MJ, Rodriguez-Fraile M, Arbizu J, et al. Contribution of time of flight and point spread function modeling to the performance characteristics of the PET/CT Biograph mCT scanner. Rev Esp Med Nucl Imagen Mol. 2013;32:13-21.

9. Panin VY, Kehren F, Michel C, Casey M. Fully 3-D PET reconstruction with system matrix derived from point source measurements. IEEE Trans Med Imaging. 2006;25:907-21.

10. Vandenberghe S, Elmbt LV, Guerchaft M, Clemental E, Verhaeghe J, Anne B, et al. Optimization of time-of-flight reconstruction on Philips GEMINI TF. Eur J Nucl Med Mol Imaging. 2009;36:1994-2001.

11. Lois C, Jakoby BW, Long MJ, Hubner KF, Barker DW, Casey $\mathrm{ME}$, et al. An assessment of the impact of incorporating time-offlight information into clinical PET/CT imaging. J Nucl Med. 2010;51:237-45.

12. Surti S, Kuhn A, Werner ME, Perkins AE, Kolthammer J, Karp JS. Performance of Philips Gemini TF PET/CT scanner with special consideration for its time-of-flight imaging capabilities. J Nucl Med. 2007;48:471-80.

13. Surti S, Karp JS. Experimental evaluation of a simple lesion detection task with time-of-flight PET. Phys Med Biol. 2009;54:373-84.

14. Conti M. Focus on time-of-flight PET: the benefits of improved time resolution. Eur J Nucl Med Mol Imaging. 2011;38:1147-57.

15. Akamatsu G, Ishikawa K, Mitsumoto K, Taniguchi T, Ohya N, Baba S, et al. Improvement in PET/CT image quality with a combination of point-spread function and time-of-flight in relation to reconstruction parameters. J Nucl Med. 2012;53:1716-22.

16. Akamatsu G, Mitsumoto K, Ishikawa K, Taniguchi T, Ohya N, Baba S, et al. Benefits of point-spread function and time of flight for PET/CT image quality in relation to the body mass index and injected dose. Clin Nucl Med. 2013;38:407-12.

17. Miyatake N, Matsumoto S, Miyachi M, Fujii M, Numata T. Relationship between changes in body weight and waist circumference in Japanese. Environ Health Prev Med. 2007;12:220-3.

18. Akamatsu G, Uba K, Taniguchi T, Mitsumoto K, Narisue A, Tsutsui Y, et al. Impact of time-of-flight PET/CT with a large axial field of view for reducing whole-body acquisition time. J Nucl Med Technol. 2014;42:101-4.

19. El Fakhri G, Surti S, Trott CM, Scheuermann J, Karp JS. Improvement in lesion detection with whole-body oncologic time-of-flight PET. J Nucl Med. 2011;52:347-53.

20. Nagaki A, Onoguchi M, Matsumoto N. Patient weight-based acquisition protocols to optimize ${ }^{18}$ F-FDG PET/CT image quality. J Nucl Med Technol. 2011;39:72-6.

21. Fukukita H, Senda M, Terauchi T, Suzuki K, Daisaki H, Matsumoto $\mathrm{K}$, et al. Japanese guideline for the oncology FDG-PET/ CT data acquisition protocol: synopsis of version 1.0. Ann Nucl Med. 2010;24:325-34.

22. Kadrmas DJ, Casey ME, Conti M, Jakoby BW, Lois C, Townsend DW. Impact of time-of-flight on PET tumor detection. J Nucl Med. 2009;50:1315-23.

23. Karp JS, Surti S, Daube-Witherspoon ME, Muehllehner G. Benefit of time-of-flight in PET: experimental and clinical results. J Nucl Med. 2008;49:462-70.

24. Akamatsu G, Mitsumoto K, Taniguchi T, Tsutsui Y, Baba S, Sasaki M. Influences of point-spread function and time-of-flight reconstructions on standardized uptake value of lymph node metastases in FDG-PET. Eur J Radiol. 2014;83:226-30.

25. Surti S, Scheuermann J, El Fakhri G, Daube-Witherspoon ME, Lim R, Abi-Hatem N, et al. Impact of time-of-flight PET on whole-body oncologic studies: a human observer lesion detection and localization study. J Nucl Med. 2011;52:712-9. 